\title{
HEMANGIOMA CAPILAR VERSUS GRANULOMA TELANGIECTÀSICO EN CAVIDAD BUCAL; UNA ARDUA LABOR DIAGNÓSTICA
}

\section{CAPILLARY HEMANGIOMATELANGIECTICUM GRANULOMA VERSUS BUCCAL CAVITY; A DIFFICULT DIAGNOSTIC TASK}

\section{TITULO CORTO: HEMANGIOMA CAPILAR VERSUS GRANULOMA TELANGIECTÀSICO}

Martha Leonor Rebolledo-Cobos ${ }^{1}$ y Manuel Vicente Escalante-Fontalvo ${ }^{1}$

\section{RESUMEN}

La consulta estomatológica puede cursar por ciertas circunstancias conflictivas en el establecimiento de diagnósticos. Cuando de tumores reactivos, hiperplásicos y proliferativos se trata, el Hemangioma Capilar (HC) y el Granuloma Telángiectasico (GT), suelen convertirse en entidades clínicas confusas debido a la semejanza clínica y al patrón de crecimiento que suelen tener; histológicamente difieren por el contenido estructural, endotelial y vascular. Estas dos lesiones son de características benignas, algunos involucionan, otros afectan estructuras vitales y en cuanto a su incidencia ambos tumores se están considerando comunes. El propósito de este artículo es evidenciar comparativamente todos los aspectos clínicos, radiográficos e histológicos del HC y el GT, por medio de un caso clínico con impresión diagnostica clínica inicial de GT y diagnostico patológico definitivo de HC.

Palabras clave: Hemangioma; Granuloma; tumor; Diagnóstico; Boca. (Fuente: DeSC)

\section{Abstract}

The dental practice may attend by certain conflicting circumstances in establishing diagnoses. When reactive, hyperplastic and proliferative tumors are treated, capillary hemangioma (HC) and telangiecticum granuloma (GT), often become confusing clinical entities because of the clinical similarity and the pattern of growth that usually have; histologically differ by structural, vascular endothelial and content. These two lesions are benign characteristics,

1. Odontólogos, especialistas en Estomatología y Cirugía Oral, Universidad de Cartagena-Colombia. Docentes Catedráticos área de cirugía bucal y semiología Universidad Metropolitana de Barranquilla. Hospital Universitario Metropolitano (Universidad Metropolitana de Barranquilla - Colombia. Correo electrónico: malereco18@gmail.com; manuelescalnte12@hotmail.com 
some regress, others affect vital structures and in terms of its impact both tumors are common considering. The purpose of this paper is comparatively demonstrate all clinical, radiographic and histological aspects of $\mathrm{HC}$ and GT, through a case diagnosed with initial clinical impression of GT and definitive pathological diagnosis of HC.

Keywords: Hemangioma; Granuloma; tumor; Diagnosis; Mouth. (Fuente:MeSH)

\section{INTRODUCCIÓN}

\section{Hemangioma Capilar}

$\mathrm{E}_{\mathrm{p}}^{1}$ hemangioma capilar es considerado una lesión proliferativa vascular benigna relativamente frecuente que puede estar presente al nacer o manifestarse durante la primera infancia. Algunos hemangiomas capilares evolucionan lentamente, su tamaño se estabiliza y persisten durante toda la vida o desaparecen lentamente. Otros pueden crecer de forma gradual pero continúan siendo benignos, alrededor del $60 \%$ a $70 \%$ de estas lesiones se encuentran en la cabeza y región del cuello ${ }^{1,2}$.

Sus características clínicas asemejan a una mora, de superficie lisa o púrpura oscuro, con o sin zonas blanquecinas relacionadas con el trauma o fricción y que suelen aumentar cuando involucionan. Su aspecto es nodular o levemente elevado. Su tamaño es variable y ocasionalmente pueden ulcerarse, estas lesiones pueden confluir cuando son varias o también presentarse como lesiones solitarias ${ }^{3}$.

El hemangioma histológicamente se clasifica en capilar y cavernoso, el primero está compuesto por pequeños vasos capilares cubiertos por una sola capa de células endoteliales apoyado en un tejido conjuntivo y estroma de densidad variable, mientras que el hemangioma cavernoso está formado por grandes vasos de paredes delgadas, o cubierto por células epiteliales separadas por tabiques con una delgada capa de tejido conectivo ${ }^{4,5}$.

La imagenología del hemangioma capilar puede indicar lesiones grandes que pueden invadir estructuras anatómicas vitales, tales como el nervio facial o la órbita. La tomografía computarizada (TC), la angiografía y la resonancia magnética (RMN) también se puede utilizar para análisis volumétrico y/o estructural de los hemangiomas y otros tumores ${ }^{6}$.

\section{Gránuloma Telángiectasico}

El granuloma telángiectasico es un tumor benigno que anteriormente se denominaba gránuloma piógeno o gravídico debido a que se presenta también en la mucosa gingival durante el embarazo o asociado con el uso de anticonceptivos orales y cambios hormonales. En la actualidad se define como una lesión benigna de la mucosa gingival a consecuencia de un trauma e irritación local, como por ejemplo exfoliación de dientes primarios, espículas óseas, trauma del cepillado, irritación gingival por placa y cálculo sobre todo cuando están localizados en encía, aparecen sin predilección de sexo y edad?

Se caracteriza clínicamente por ser exofítico en ocasiones pediculado o sésil y de consistencia blanda, la superficie puede ser lisa, granular o lobulada debido a los espacios vasculares superficiales dilatados que producen una apariencia de mora semejante al hemangioma capilar (Tabla 1$)^{7}$.

La descripción histológica que reportan algunos autores va relacionadas a características de neo-formación vascular con proliferación y engrosamiento del epitelio escamoso ${ }^{8}$. En cuanto a sus características radiográficas se asemejan a una erosión del hueso o pérdidaósea en la zona afectada, en ocasiones con o sin desplazamiento de órganos dentales ${ }^{9}$.

Las terapéuticas tanto para el hemangioma capilar y el granuloma telángiectasico, son variables, se debe individualizar en cada caso; dependiendo del tamaño de la lesión y el involucro a otras estructuras, ya que podría afectar la estética u órganos y sistemas. Estos tratamientos incluyen: escleroterapia, terapia láser o la terapia combinada, también es opción de tratamiento la biopsia escisional, crioterapia entre otras ${ }^{10,7}$.

Es indispensable el conocimiento por parte de los especialistas de la estomatología y la cirugía oral, de las 
diferencias y semejanzas existentes entre los tumores de contenido vascular, con el fin de establecer terapéuticas acertadas que eviten al máximo el riesgo procedimental y la recidiva de la lesión.

Tabla 1. Cuadro comparativo entre hemangioma capilar y granuloma telángiectasico

\begin{tabular}{|c|c|c|}
\hline & $\begin{array}{c}\text { Hemangioma } \\
\text { Capilar }\end{array}$ & $\begin{array}{l}\text { Gránuloma } \\
\text { Telángiectasico }\end{array}$ \\
\hline $\begin{array}{l}\text { Aparición al } \\
\text { nacimiento }\end{array}$ & $30 \%$ & $0 \%$ \\
\hline $\begin{array}{l}\text { Relación } \\
\text { hombre/mujer }\end{array}$ & $2: 1$ & $1: 1$ \\
\hline Incidencia & $30 \%$ & $30 \%$ \\
\hline $\begin{array}{l}\text { Crecimiento y } \\
\text { clínica }\end{array}$ & $\begin{array}{c}\text { Crecimiento } \\
\text { lento, } \\
\text { involución y } \\
\text { Crecimiento } \\
\text { proporcional, } \\
\text { Color rojo/ } \\
\text { violáceo/ } \\
\text { azulado, } \\
\text { sésil y/o } \\
\text { pediculado. }\end{array}$ & $\begin{array}{l}\text { Crecimiento rápido, } \\
\text { no involuciona. } \\
\text { Color rojo/ } \\
\text { violáceo, sésil } \\
\text { y/o pediculado, } \\
\text { sangrante. }\end{array}$ \\
\hline Histología & $\begin{array}{l}\text { Proliferación } \\
\text { endotelial, } \\
\text { malformación } \\
\text { vascular. }\end{array}$ & $\begin{array}{l}\text { Proliferación } \\
\text { fibrocolágena y } \\
\text { formación vascular } \\
\text { con infiltrado } \\
\text { inflamatorio } \\
\text { crónico. }\end{array}$ \\
\hline Terapéutica & $\begin{array}{l}\text { Crioterapia, } \\
\text { escleroterapia, } \\
\text { laserterapia, } \\
\text { escisión. }\end{array}$ & $\begin{array}{l}\text { Escisión, } \\
\text { crioterapia. }\end{array}$ \\
\hline Pronostico & Favorable & Favorable \\
\hline
\end{tabular}

Fuente: elaboración propia basada en los autores1-7, 9-10.

\section{CASO CLÍNICO}

Se evidencia un paciente masculino de 56 años de edad, natural y procedente Barranquilla, Atlántico-Colombia, sin antecedentes medico-personales de relevancia clínica, el cual acude a la clínica odontológica de la Fundación Hospital Universitario Metropolitano (FHUM), por presentar lesión tumoral de aproximadamente 11 x $10 \mathrm{~mm}$ de diámetro en el maxilar superior a nivel del segundo y tercer molar izquierdo, con relación al fondo del surco, color rojizo/violáceo con zonas hiperqueratósicas blanquecinas y sin llegar a producir asimetría facial, asintomático de evolución desconocida por el paciente (Figura 1).

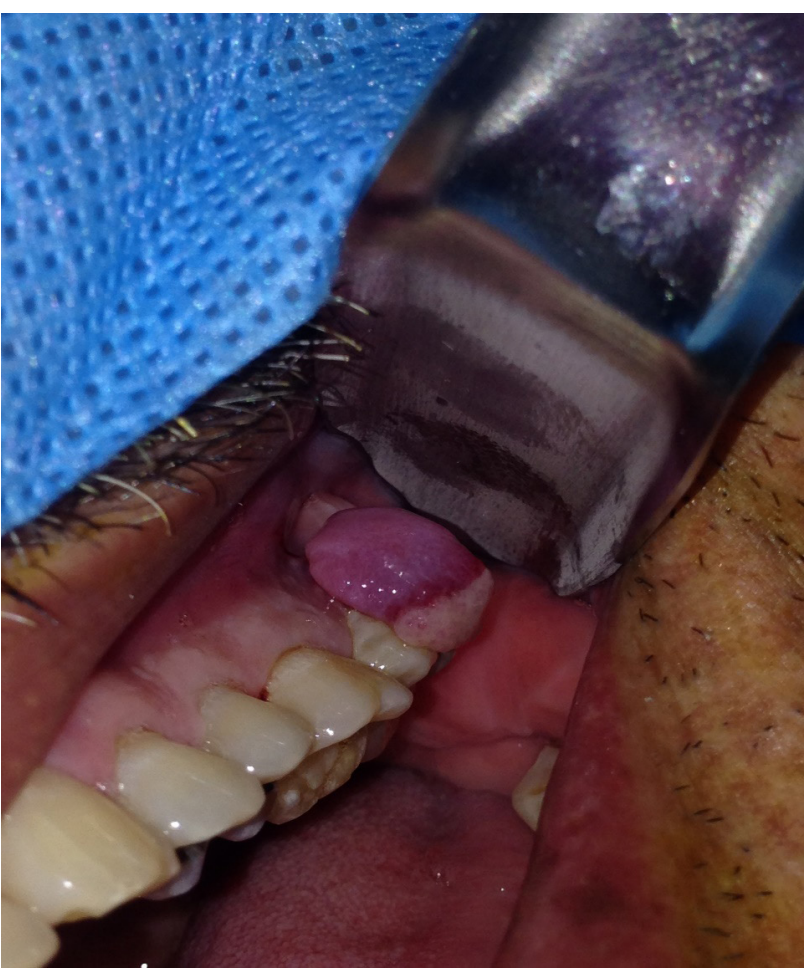

Figura 1. Imagen clínica del hemangioma capilar oral

Al examen radiográfico no se evidencia ninguna lesión en el sitio anteriormente mencionado, los órganos dentales involucrados se evidenciaron sanos clínica y radiográficamente, estableciéndose impresiones diagnosticas de granuloma telángiectasico; para lo cual finalmente se decidió realizar escisión quirúrgica. Seguidamente, se le informa al paciente el plan de manejo a ejecutar, autorizando e indicando riesgos y consecuencias; firmando un consentimiento informado, que incluye su autorización para la toma de fotografías y la presente publicación. Las impresiones diagnosticas iníciales involucraron gránuloma periférico de células 
gigantes, fibroma periférico y hemangioma capilar. El espécimen extraído, fue enviado a dos patólogos diferentes, desconocidos entre sí, con el fin de aclarar dudas con respecto al diagnóstico presuntivo. Los reportes patológicos manifestaron una confluencia de criterios semejantes a las características de un hemangioma capilar, los cuales mostraban presencia de un recambio de células endoteliales e incremento en el número de mastocitos, acompañado de un estroma de tejido conjuntivo de sostén (Figura 2 y 3 ).

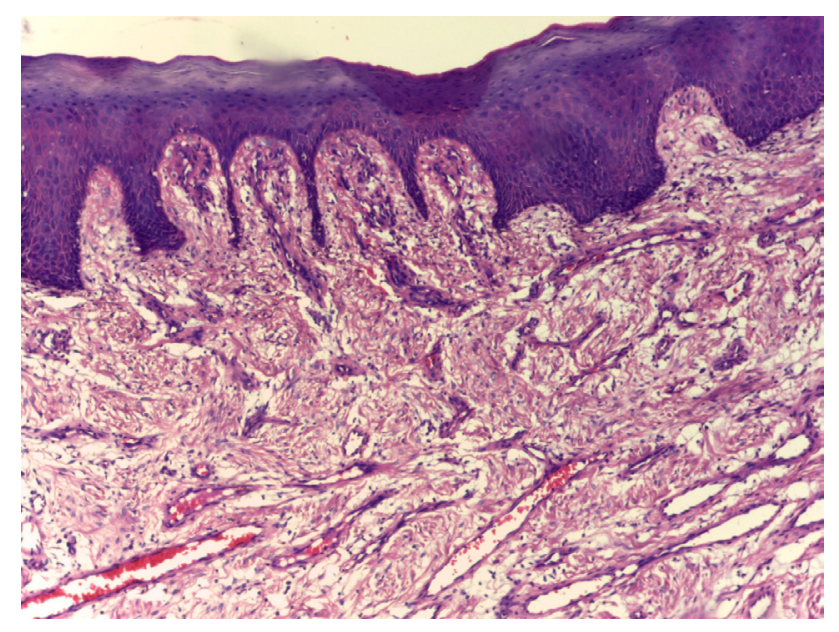

Figura 2. Imagen histopatológica con tinción fucsinaeosina a $400 \mathrm{X}$ que evidencia diagnóstico definitivo de hemangioma capilar oral.

\section{0}

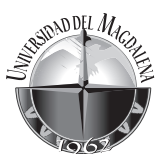

\section{DISCUSIÓN}

Los hemangiomas constituyen el $7 \%$ de todos los tumores benignos en la infancia y la niñez, siendo relativamente rara la aparición en cavidad oral; suele ser una entidad patológica que genera fácil confusión con otros tumores benignos de mayor prevalencia como lo es el granuloma periférico de células gigantes y el granuloma telángiectasico, en semejanza con nuestro caso la impresión clínica se atribuía más en relación a un granuloma, pero se contemplaba la presunción por las características clínicas que fuera una lesión exclusivamente vascular ${ }^{4}$. Estas características clínicas hacen referencia al sangrado a la palpación, al mínimo roce con la masticación y a la ejecución de cualquier movimiento ${ }^{7}$.

El hemangioma capilar posee una naturaleza de crecimiento y desarrollo, mientras que el granuloma telángiectasico posee etiologías multifactoriales, trauma, cuerpos extraños u otros factores adquiridos; en discrepancia con el presente caso, la lesión no poseía una etiología definida y el paciente desconocía la evolución ${ }^{11}$. Un evento que llama la atención es la denominación por diversos autores a la lesión de un "hemangioma capilar, como un tipo granuloma" o "hemangioma capilar lobular", asemejando al conflicto diagnostico presentado en este artículo, donde lo consideramos como una ardua labor diagnostica. Puede ser difícil hacer una diferenciación microscópica entre un verdadero hemangioma de la infancia y un granuloma telángiectasico, sin embargo, este último exhibe diferencias de inmuno y ultra estructurales muy diferentes a un hemangioma, como muestra el presente caso, donde el informe que emite el patólogo se encamina exclusivamente a caracterizar un hemangioma ${ }^{11-13}$.

La aparición clínica de ese tipo de tumores benignos puede llevar a un diagnóstico y tratamiento erróneo de otra lesión más grave, debido a las malformaciones vasculares, los riesgos quirúrgicos tienden a ser considerables. El diagnóstico diferencial puede incluir el carcinoma de células escamosas, sarcoma de Kaposi, carcinoma metastásico y una lesión benigna como angiomátosis bacilar, fibroma periférico entre otras lesiones vasculares. Por lo tanto, se concluye que el diagnostico partiendo de la semiología de estos tumores en semejanza, se convierten en todo un reto $0^{4,7,13}$. 
Se obtuvo el consentimiento informado por parte del paciente para la utilización y publicación de la información acompañada de imágenes con fines académicos y científicos.

\section{AGRADECIMIENTOS}

Diana Cristina Castro Buitrago y Jairo Better, estudiantes de último año de odontología, Universidad Metropolitana de Barranquilla, Colombia.

\section{REFERENCIAS BIBLIOGRÁFICAS}

1. Castro CF, Raimondi R, Martinez-Carvajal W, Martinez-Nina S. Tumor benigno de tejido blando oral "Hemangiomaoral": A propósito de un caso. Rev MédCient "Luz Vida". 2011; 2(1): 51-54.

2. Okoji VN, Alonge TO, Olusanya AA. Intra-tumoral ligation and the injection of sclerosant in the treatment of lingual cavernous hemangioma. Niger J Med. 2011; 20: $172-5$.

3. Phan, Tai Anh; Adams, Susan; Wargon, Orli. Segmental hemangiomas of infancy: a review of 14 cases. AustralasianJournal of Dermatology, 2006 Nov; 47(4): 242-247.

4. Kamala KA, Ashok L, Sujatha GP. Cavernous hemangioma of the tongue: A rare case report. ContempClin Dent. 2014 Jan-Mar; 5(1): 95-98.

5. Neville BW, Damm DD, Allen CM, Bouquet JE. Abnormalities of teeth. In: Oral and maxillofacial pathology. 2nd ed. Oxford, UK: W.B. Saunders Company; 2002.

6. Bonet-Coloma C, Mínguez-Martínez I, Palma-Carrió C, Galan-Gil S, Penarroche-Diago M, Minguez-SanzJM. Clinical characteristics, treatment and outcome of 28 oral hemangiomas in pediatric patients.Med Oral PatolOralCirBucal. 2011; 16: e19-22.

7. Rebolledo-Cobos M, Harris-Ricardo J, Cantillo-Pallares O, Carbonell-Muñoz Z, Díaz Caballero A. Granuloma telangiectásico en cavidad oral. AvOdontoestomatol. 2010 Oct; 26(5): 249-253.

8. Díaz-Caballero AJ, Vergara-Hernández CI, CarmonaLorduy M. Granuloma telangiectásico en cavidad oral. Reporte de un caso clínico. AvOdntoestomatol 2009 junio; 25 (3): 131-135.

9. Aguilera FA, Shalkow KJ, de la Teja AE, Durán GA. Criterios estomatológicos para el tratamiento del paciente con anomalías vasculares. Informe de cuatro casos. Acta PediatrMex 2009; 30(5): 247-53

10. Newman J, Anand V. Applications of thediode laser in otolaryngology. Ear NoseThroat J 2002; 81: 850-851.

11. Murthy J. Vascular anomalies. Indian J Plst Surg. 2005; 38: 56-62.

12. Mulliken JB. Cutaneous Vascular Anomalies. Tumors of Head and Neck and Skin. Philadelphia: Vol. 5 B Saunders Company Ltd; 1990.

13. Rebolledo-Cobos M, Cantillo-Payares O, Díaz-Caballero A. Fibroma periférico odontogénico: A propósito de un caso. AvOdontoestomatol. 2010 Ago; 26(4): 183-187.

Para citar este artículo: Rebolledo-Cobos M, Escalante-Fontalvo M. Hemangioma Capilar versus Granuloma Telangiectàsico en cavidad bucal; una ardua labor diagnóstica. Duazary. 2015 dic; 12 (2): 187 - 191 